\title{
The triphibious warfare against viruses
}

\author{
William J. Liu ${ }^{1 *} \&$ Di Liu ${ }^{2,3 *}$ \\ ${ }^{1}$ Key Laboratory of Medical Virology and Viral Diseases, Ministry of Health of People's Republic of China, National Institute for Viral Disease \\ Control and Prevention, Chinese Center for Disease Control and Prevention, Beijing 102206, China; \\ ${ }^{2}$ CAS Key Laboratory for Pathogenic Microbiology and Immunology, Institute of Microbiology, Chinese Academy of Sciences, Beijing \\ 100101, China; \\ ${ }^{3}$ Computational Virology Group, CAS Key Laboratory of Special Pathogens and Biosafety, Wuhan Institute of Virology, Chinese Academy of \\ Sciences, Wuhan 430071, China
}

Received November 16, 2017; published online December 1,2017

Citation: Liu, W.J., and Liu, D. (2017). The triphibious warfare against viruses. Sci China Life Sci 60, 1295-1298. https://doi.org/10.1007/s11427-017-9252-y

Despite the long journey undertaken by mankind to confront the diseases caused by viruses, people were unaware of the existence of viruses until the closing years of the 19th century, when the Russian biologist Dmitri Ivanovsky demonstrated that tobacco plants could be infected by a toxin smaller than bacteria, followed by which the Dutch microbiologist Martinus Beijerinck termed the toxin as a contagium vivum fluidum (soluble living germ) and re-introduced the word "virus" (derived from the Latin word "virus" meaning poison; the modern meaning is an "agent that causes infectious disease" that was first recorded in 1728). Later on, thousands of virus species infecting animals, plants, and bacteria were discovered, especially in the second half of the 1900 s with the development of virus isolation techniques. In recent decades, the emerging and re-emerging human-infecting viruses have been reported to represent a huge threat to public health and have become a global concern. Different elements may drive the current trends in emerging and re-emerging infectious diseases, which are as follows: (i) the contact patterns between human beings, their commensal organisms, and wildlife reservoirs are changing due to human behaviors, (ii) the interaction between viruses and their potential hosts and vectors, such as bats and mosquitoes, may cause changes in

*Corresponding author (William J. Liu, email: liujun@ivdc.chinacdc.cn; Di Liu, email: liud@im.ac.cn) richness and abundance, and (iii) the transmission gap of the viruses between people becomes narrow due to urbanization and globalization (Olival et al., 2017).

Field surveillance and laboratory investigation comprise the two primary forefronts of the combat against viruses. The former activity involves hunting of the virus in humans and animal reservoirs, the molecular ecology, and emergency disposal, and the latter comprises investigations on the pathogenesis, host immunity, and drug and vaccine development. This thematic issue is dedicated to the current activities of the studies covering these strategies, which may provide an efficient network for controlling these emerging and re-emerging viruses.

In fact, since the conquer of SARS-CoV, China has made great improvements in the prevention and emergency systems against infectious disease outbreaks. Han et al. have provided insights into the national strategies of China, especially the surveillance system, that help China transit from a passive to a proactive role to fight against infectious diseases (Han et al., 2017). The surveillance system has collected professional forces from different institutes and laboratories covering health, agriculture, and military sectors and universities and hospitals (Han et al., 2017). The Chinese Academy of Sciences (CAS) has been one of the backbone forces during this process and has been playing essential roles in the fight against infectious diseases since 2005, when the H5N1 sub- 
type, a highly pathogenic avian influenza virus (HPAI), was identified among wild birds in Qinghai Lake (Liu et al., 2005). The CAS Center for Influenza Research and Early-warning (CASCIRE) was established in 2014 with the aim of developing a coordinated emergency response and research capacity on the emerging or re-emerging infectious diseases (Bi et al., 2017).

The continuously emerging and re-emerging avian influenza viruses, including H5N1, H5N6, H7N9, and H9N2, have been particular concerns in terms of public health. Of note, the H7N9 virus has underwent five epidemic waves in China since its emergence in March 2013 (Liu et al., 2014). The fifth wave in the 2016-2017 flu season was characterized as the largest epidemic causing the highest number of human cases and the emergence of HPAI H7N9 in both humans and poultry (Deng et al., 2017). In this issue, Tang et al. reviewed the epidemiology, evolution, vaccine development, drug resistance, and alternative drug development of the five epidemic waves of H7N9 (Tang et al., 2017). Xiao et al. analyzed the efficacy of synthetic peptides that could enhance the immune responses induced by inactive viral vaccines against avian influenza viruses. The vaccine candidates combined with the peptide adjuvant may not only effectively inhibit the spread of avian influenza viruses in poultry but also control the transmission of the virus from poultry to humans (Xiao et al., 2017).

The majority of emerging infectious diseases in human beings are zoonotic, which indicates the fundamental role of wildlife, especially rodents and bats, as the reservoirs of emerging viruses (Olival et al., 2017). Ao et al. identified two new divergent bocaparvoviruses in wild Himalayan marmots in the Qinghai-Tibetan Plateau, China, during 2013-2014 (Ao et al., 2017). Obameso et al. conducted a 2-year follow-up study of a special bat population in a cave of south China and showed that a previously identified new bat-derived coronavirus, Ro-BatCoV GCCDC1, persistently circulated among the bats (Obameso et al., 2017).

The surveillance of viruses among natural vectors is also an important work, considering the recent global spread of arthropod-borne viruses such as Zika virus and dengue virus. Zika virus was first isolated by $\mathrm{Fu}$ et al. in Mainland China from two different species of mosquitoes, namely, Culex pipiens quinquefasciatus and Armigeres subbalbeatus (Fu et al., 2017). Song et al. identified a Banna virus (genus eadornavirus, family Reoviridae) from Culicoides collected in the Sino-Burmese border region of Yunnan Province, China, which indicates that Culicoides can also be a vector for Banna virus and that the virus does not have a species barrier between the two blood-sucking insects Culicoides and mosquitoes (Song et al., 2017).

The accumulation of massive genome data based on next-generation sequencing of the emerging and re-merging viruses provides an opportunity for conducting a pathogen genomic surveillance to elucidate the origin, transmission, and evolution of emerging viruses. However, this also requires a series of advanced and standard bioinformatics tools in the field of emerging pathogens. Shi et al. reviewed the mode of application of pathogen genomics in the surveillance of recently emerging viral pathogens in China (Shi et al., 2017). Wei et al. utilized H7N9 avian influenza viruses as an exemplified application to establish the platform of Bayesian evolutionary analysis for studying emerging infectious diseases (Wei et al., 2017).

Immune memory in the hosts generally provides an efficient protection against secondary viral infections. However, with the emerging or re-emerging of the viruses that are genetically related to previously prevalent viruses, the cross-reactivity of the pre-existing immune memory to the secondary virus may lead to either a cross-protection or an antibody-dependent enhancement. This phenomenon causes complications in vaccine design and testing. Li et al. assessed the in vitro enhancement of Zika virus infectivity by a series of human monoclonal antibodies and provided the first demonstration of a quantitative association between the concentrations for neutralization and peak enhancement for the same antibody (Li et al., 2017).

Zeng et al. demonstrated that the majority of SARS-CoV receptor-binding domain (RBD)-specific antibodies could cross-neutralize the SARS-like coronavirus strain WIV1, but not SHC014, due to the minor genetic distinction on the RBDs of the two viruses, although they share the same receptor ACE2 as that of SARS-CoV (Zeng et al., 2017). Zhao et al. summarized the current updates of the characteristics of human T-cell immunological responses against the recently emerging or re-emerging viruses, such as H7N9 influenza virus, Zika virus, MERS-CoV, and Ebola virus, which may provide useful recommendations for clinical diagnosis and vaccine development (Zhao et al., 2017).

Structural investigations on the emerging and re-emerging viruses can provide a visualized insight into the pathogenesis of the viruses and also shed light on the targets for drug development. In this issue, Wang et al. reported the first crystal structure of the C-terminal fragment of YFV NS1, a key player in the replication, pathogenesis, and immune evasion of YFV. Their work enriches our knowledge on the characteristics of flaviviral NS1 proteins and offers a new guide for the development of new drug candidates (Wang et al., 2017).

In summary, the triphibious warfare against viruses appears to be endless, considering the continuous emergence of new viruses and also the mutations of the existing viruses. However, the development of new techniques based on the most recent innovations has increased our confidence in controlling and preventing the emerging infectious diseases in a more efficient manner. The final objective might only be to minimize the impact of any epidemics on the economy and social development by controlling them during the initial stages. 
Compliance and ethics The author(s) declare that they have no conflict of interest.

Ao, Y., Li, X., Li, L., Xie, X., Jin, D., Yu, J., Lu, S., and Duan, Z. (2017). Two novel bocaparvovirus species identified in wild Himalayan marmots. Sci China Life Sci 60, 1348-1356.

Bi, Y., Shi, W., Chen, J.,Chen, Q., Ma, Z.,Wong, G., Tian, W., Yin, R., Fu, G., Yang, Y., Wang, Q., He, S., Li, X., Xia, Q., Wang, L., Pan, Z., Li, L., Li, H., Xu, W., Luo, Y., Zeng, H., Dai, L., Li, X., Liu, Y., Lei, F., Liu, W., and Gao, G.F. (2017). CASCIRE surveillance network and works on avian influenza viruses. Sci China Life Sci 60, 1386-1391.

Deng, Y., Li, C., Han, J., Wen, Y., Wang, J., Hong, W., Li, X., Liu, Z., Ye, Q., Li, J., Zhou, C., Yu, L., Qin, C., Zhang, F., and Jiang, T. (2017). Phylogenetic and genetic characterization of a 2017 clinical isolate of H7N9 virus in Guangzhou, China during the fifth epidemic wave. Sci China Life Sci 60, 1331-1339.

Fu, S., Song, S., Jia, C., Zhang, J., Liu, H., Li, Y., Li, X., Gao, X., Xu, Z., Liu, G., Liu, G., Wang, D., Tian, Z., Zhou, J., He, Y., Lei, W., Wang, H., Wang, B., Lu, X., and Liang, G. (2017). ZIKA virus isolated from mosquitoes: a field and laboratory investigation in China. Sci China Life Sci 60, 1364-1371.

Han, M., Gu, J., Gao, G.F., and Liu, W.J. (2017). China in action: national strategies to combat against emerging infectious diseases. Sci China Life Sci 60, 1383-1385.

Li, M., Wang, X., Wang, Q., Yu, L., Wang, L., Yan, J., Zhang, F., Zhang, L., Gao, G.F., and Jin, X. (2017). Both structure and function of human monoclonal antibodies contribute to enhancement of Zika virus infectivity in vitro. Sci China Life Sci 60, 1396-1398.

Liu, J., Xiao, H., Lei, F., Zhu, Q., Qin, K., Zhang, X.W., Zhang, X.L., Zhao, D., Wang, G., Feng, Y., Ma, J., Liu, W., Wang, J., and Gao, G.F. (2005). Highly pathogenic $\mathrm{H} 5 \mathrm{~N} 1$ influenza virus infection in migratory birds. Science 309, 1206-1206.

Liu, J., Xiao, H., Wu, Y., Liu, D., Qi, X., Shi, Y., and Gao, G.F. (2014). H7N9: a low pathogenic avian influenza A virus infecting humans. Curr Opin Virol 5, 91-97.
Obameso, J.O., Li, H., Jia, H., Han, M., Zhu, S., Huang, C., Zhao, Y., Zhao, M., Bai, Y.,Yuan, F., Zhao, H., Peng, X., Xu, W., Tan, W., Yuen, K-Y., Zhao, Y., Liu, W.J., Lu, L., and Gao, G.F. (2017). The persistent prevalence and evolution of cross-family recombinant coronavirus GCCDC1 among a bat population: a two year follow-up. Sci China Life Sci 60, 1357-1363.

Olival, K.J., Hosseini, P.R., Zambrana-Torrelio, C., Ross, N., Bogich, T.L., and Daszak, P. (2017). Host and viral traits predict zoonotic spillover from mammals. Nature 546, 646-650.

Shi, Q., Li, J., Zhou, H., and Gao, G.F. (2017). Pathogen genomic surveillance elucidates the origins, transmission and evolution of emerging viral agents in China. Sci China Life Sci 60, 1317-1330.

Song, S., Li, Y., Fu, S., Lei, W., Guo, X., Feng, Y., Gao, X., Li, X., Yang, Z., Xu, Z., He, Y., Wang, H., Zhou, H., Wang, B., Lu, X., and Liang, G. (2017). Genome sequencing and phylogenetic analysis of Banna virus (genus Seadornavirus, family Reoviridae) isolated from Culicoides. Sci China Life Sci 60, 1372-1382.

Tang, J., and Wang, D. (2017). Research progress in human infection with avian influenza H7N9 virus. Sci China Life Sci 60, 1299-1306.

Wang, H., Han, M., Qi, J., Hilgenfeld, R., Luo, T., Shi, Y., and Gao, G.F. (2017). Crystal structure of the C-terminal fragment of NS1 protein from yellow fever virus. Sci China Life Sci 60, 1403-1406.

Wei, X., Chen, M., and Cui, J. (2017). Bayesian evolutionary analysis for emerging infectious disease: an exemplified application for H7N9 avian influenza viruses. Sci China Life Sci 60, 1392-1395.

Xiao, J., Zhang, L., Wang, Z., Xiang, W., Lu, P., Zhao, Y., Han, M., Ma, A., Qi, P., Wang, M., Gao, G.F., and Liu, W.J. (2017). Conserved peptides enhance immune efficiency of inactive vaccines against emerging avian influenza viruses in chicken. Sci China Life Sci 60, 1340-1347.

Zeng, L.P., Ge, X.Y., Peng, C., Tai, W., Jiang, S., Du, L., and Shi, Z.L. (2017). Cross-neutralization of SARS coronavirus-specific antibodies against bat SARS-like coronaviruses. Sci China Life Sci 60, 1399-1402.

Zhao, M., Zhang, H., Liu, K., Gao, G.F., and Liu, W.J. (2017). human T-cell immunity against the emerging and re-emerging viruses. Sci China Life Sci 60, 1307-1316. 

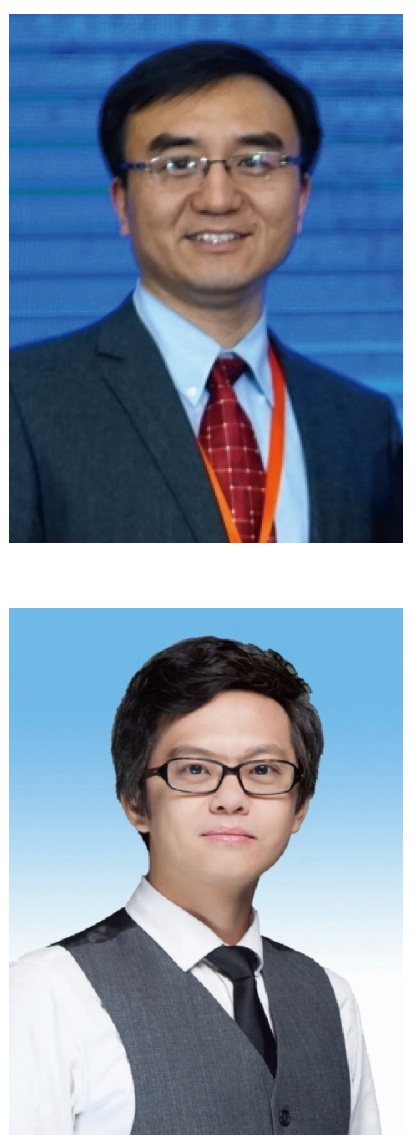

\section{Biographical Sketch}

Dr. William J. Liu was born in Inner Mongolia, China, and graduated from the School of Public Health, Peking University Health Science Center, in 2005. He received his Ph.D. degree in molecular biology and biochemistry from the Institute of Microbiology, Chinese Academy of Sciences, in 2010. Later on, he underwent postdoctoral training at the Institute of Biophysics, Chinese Academy of Sciences in China, and the Yale School of Medicine in the US. He is currently a fulltime professor at the National Institute for Viral Disease Control and Prevention, Chinese Center for Disease Control and Prevention. The primary research focus of his group is the prevention and control of influenza viruses and T-cell recognition.

Dr. Di Liu was born in Beijing, China, and graduated from School of Life Sciences, Peking University, in 2000. He received his Ph.D. degree in bioinformatics from the Centre for Bioinformatics (CBI), Peking University, in 2005. Later on, he worked in the Institute of Microbiology, Chinese Academy of Sciences, as a postdoctoral fellow, assistant professor, associate professor, and professor (2014). He was awarded the Top-notch Young Professionals. Currently, he leads a group focusing on computational virology at Wuhan Institute of Virology, Chinese Academy of Sciences. His research interests cover the genomes and evolution of pathogens. 